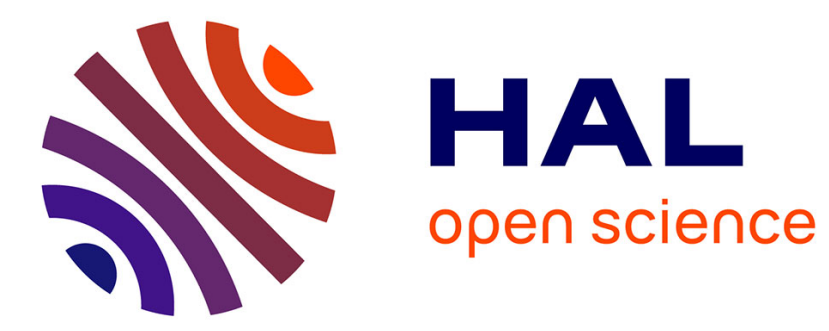

\title{
Coherent microwave-to-optical conversion by three-wave mixing in a room temperature atomic system
}

K. V Adwaith, Asha Karigowda, Charudatta Manwatkar, Fabien Bretenaker, Andal Narayanan

\section{- To cite this version:}

K. V Adwaith, Asha Karigowda, Charudatta Manwatkar, Fabien Bretenaker, Andal Narayanan. Coherent microwave-to-optical conversion by three-wave mixing in a room temperature atomic system. Optics Letters, 2019, 44 (1), pp.33. 10.1364/OL.44.000033 . hal-02107656

\section{HAL Id: hal-02107656 \\ https://hal.science/hal-02107656}

Submitted on 23 Apr 2019

HAL is a multi-disciplinary open access archive for the deposit and dissemination of scientific research documents, whether they are published or not. The documents may come from teaching and research institutions in France or abroad, or from public or private research centers.
L'archive ouverte pluridisciplinaire HAL, est destinée au dépôt et à la diffusion de documents scientifiques de niveau recherche, publiés ou non, émanant des établissements d'enseignement et de recherche français ou étrangers, des laboratoires publics ou privés. 


\title{
Coherent microwave-to-optical conversion by three-wave mixing in a room temperature atomic system
}

\author{
K. V. Adwaith, ${ }^{1}$ Asha Karigowda, ${ }^{1,2}$ Charudatta Manwatkar, ${ }^{1}$ Fabien Bretenaker, ${ }^{1,3}$ [(] \\ and Andal Narayanan ${ }^{1, *}$ \\ ${ }^{1}$ Light and Matter Physics Group, Raman Research Institute, Bangalore 560080, India \\ ${ }^{2}$ Department of Physics, Kuvempu University, Shankarghatta, Shivamogga 577451, India \\ ${ }^{3}$ Laboratoire Aime Cotton, CNRS, Universite Paris-Sud 11, ENS Cachan, 91405 Orsay Cedex, France \\ *Corresponding author: anda/@rri.res.in
}

Received 17 October 2018; accepted 14 November 2018; posted 19 November 2018 (Doc. ID 348576); published 17 December 2018

\begin{abstract}
We experimentally observe coherent generation of a nearinfrared optical field through a three-wave mixing phenomenon in an atomic energy level scheme of ${ }^{85} \mathrm{Rb}$ atoms. This nonlinear generation process in a centro-symmetric thermally broadened atomic system is made possible through a novel interaction between induced electric and magnetic dipoles. The two-photon and three-photon coherence present in our scheme eliminates excited state decoherence. Thus, our scheme represents a minimal optical decoherence scheme which could be used to transfer quantum states between microwave-to-optical frequency regimes with nearunit fidelity. () 2018 Optical Society of America
\end{abstract}

https://doi.org/10.1364/OL.44.000033

Nonlinear frequency conversion from microwave-to-optical frequencies has garnered a lot of attention in the past decade [1-3]. This is due to the ease of transport and detection of gigahertz signals through optical channels and the essentially noisefree nature of the frequency conversion process. The latter attribute has been central to proposing high-fidelity classical and quantum conversion of signals between microwave and optical frequencies [4,5]. A range of devices has been proposed to demonstrate this frequency conversion phenomenon. Starting from the commonly used electro-optic-modulator, several authors have investigated carefully engineered whisperinggallery-mode resonators [6,7], micro- and nano-sized hybrid electro-opto-mechanical devices operating at a few kelvin [8,9], rare-earth doped crystals [10] and gaseous atomic systems with induced coherence [11] as viable architectures for effecting nonlinear frequency generation and signal transfer. In atomic systems, there have been several theoretical proposals [12-15], but very few experimental demonstrations [16]. These studies highlight various aspects of the nonlinear generation phenomenon such as efficiency, bi-directionality, and non-reciprocity.

A four-wave mixing phenomenon has been a widely utilized nonlinear frequency generation process in atomic systems $[17,18]$. Beginning with lasing without inversion [19], experiments showing new frequency generation have been performed, which combine atomic coherence effects with a four-wave-mixing process [20-23]. In this Letter, we demonstrate a novel three-wave mixing process in a room temperature gaseous atomic vapor of ${ }^{85} \mathrm{Rb}$ atoms, which utilizes induced atomic coherence to generate a near-infrared optical field. This three-wave mixing process combines an electric dipole and a magnetic-dipole transition in optical and microwave frequency domains, respectively, leading to the generation of a new infrared frequency.

A unique feature of our system is that despite thermal motion of our atomic ensemble at room temperature and despite resonant nonlinear interaction, the generation process is coherent. It is not affected by the Doppler width or by the decay processes from the excited states. This is due to the fulfillment of both two-photon and three-photon resonance conditions for our system by all the three fields. During the generation process of the probe field, the probe and the coupling satisfy a twophoton resonance that shelves the atoms in a dark state due to the electromagnetically induced transparency effect [24], making the process immune to noise effects due to spontaneous decay from excited states. As is well known, the two-photon resonance condition is Doppler free. This Doppler-free condition remains valid, even during the fulfillment of the threephoton resonance condition between the coupling, microwave, and the generated probe fields due to the smallness of the Doppler width for microwaves.

Thus, due to a nonlinear interaction between induced electric and magnetic dipoles and due to the formation of atomic ground state coherence, we have demonstrated for the first time, to the best of our knowledge, the lowest-order coherent nonlinear frequency conversion process from an atomic ensemble. The bandwidth for coherent conversion from microwaveto-optical frequencies with this scheme is shown to be about $825 \mathrm{kHz}$. The efficiency of our conversion process is about $2.6 \times 10^{-4} \%$. The efficiency is mainly dictated by the weakness of a magnetic-dipole interaction induced by the microwave field. Nevertheless, the fidelity that could be achieved by our scheme for signal transfer will be very high due to the absence of major sources of decoherence in our conversion 
mechanism. This aspect can be exploited, especially for transfer of quantum signals, as previously shown [25].

In our experiment, the optical coupling beam is derived from an external cavity diode laser with a linewidth of a few hundred kilohertz. This coupling $\left(\omega_{\mathrm{c}}\right)$ beam connects the meta-stable ground state $5 S_{1 / 2}, F=3$ state $(|2\rangle)$ to the excited state $5 P_{1 / 2}, F=3^{\prime}(|3\rangle)$ in the D1 transition manifold of ${ }^{85} \mathrm{Rb}$, as shown in Fig. 1. The detuning of the coupling field from the $|2\rangle \rightarrow|3\rangle$ transition is denoted by $\delta_{\mathrm{c}}$. The $|2\rangle$ state is also connected to the ground state $5 S_{1 / 2}, F=2$ state $(|1\rangle)$ through a microwave drive field $\omega_{\mu \mathrm{w}}$ obtained from a microwave source, as shown in Fig. 2. The detuning of the drive field from the $|1\rangle \rightarrow|2\rangle$ transition is denoted by $\delta_{\mu \mathrm{w}}$. Since an electric-dipole transition is forbidden between states $|1\rangle$ and $|2\rangle$, this microwave field drives a magnetic-dipole transition. A vapor cell of a length of $5 \mathrm{~cm}$ containing enriched ${ }^{85} \mathrm{Rb}$ atoms at a temperature of $300 \mathrm{~K}$ is used in our experiment. To overcome the weakness of the magnetic-dipole transition driven by the microwave field, the vapor cell is placed inside a cylindrical high-Q microwave cavity. This cavity supports a $\mathrm{TE}_{011}$ mode of the microwave field with a high quality factor of about 9000 at $3.0357 \mathrm{GHz}$ which is the separation between the ground hyper-fine levels. This is shown in the schematic of the experimental setup, also given in Fig. 2.

A typical experimental run consists of locking the coupling laser at resonance with $\delta_{\mathrm{c}}=0$. The microwave drive field is then scanned around its resonance with $\delta_{\mu \mathrm{w}}$ spanning through

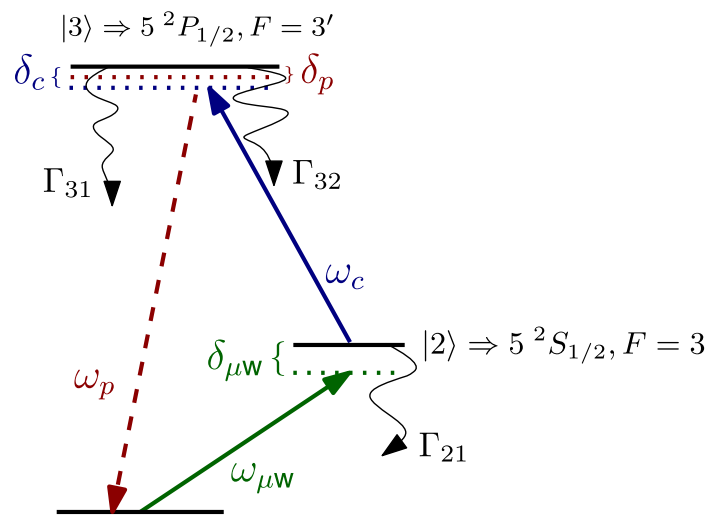

$|1\rangle \Rightarrow 5^{2} S_{1 / 2}, F=2$

Fig. 1. $\Delta$ System formation using hyper-fine levels of ${ }^{85} \mathrm{Rb}$ in its D1 manifold.

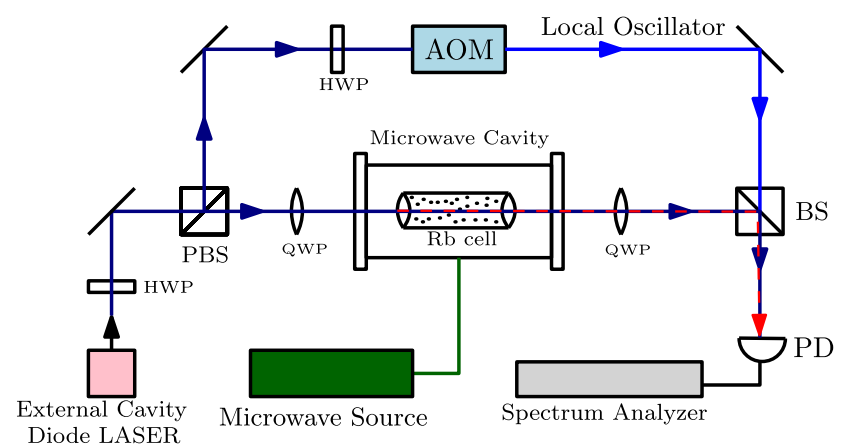

Fig. 2. Experimental setup. $\pm 2 \mathrm{MHz}$. During this scan, the coupling optical field and the microwave drive field interact nonlinearly with the atoms. A sum-frequency generation takes place, giving rise to a probe field $\omega_{\mathrm{p}}$ with a frequency $\omega_{\mathrm{p}}=\omega_{\mathrm{c}}+\omega_{\mu \mathrm{w}}$. In our experiment, the phase-matching requirement $\vec{k}_{p}=\vec{k}_{c}+\vec{k}_{\mu w}$ for efficient generation ensures that the generated infrared probe field is in the same direction as the undepleted coupling field. The coupling and probe beams exiting the cavity are then mixed with a local oscillator reference beam derived from the coupling laser (Fig. 2), and a heterodyne detection of the generated probe field is made in a spectrum analyzer.

We now proceed to show the characteristics of the generated probe field. The spectral profile of the generated probe field is shown in Fig. 3 when both the coupling and microwave fields are at resonance with their respective transitions. This spectral profile is a beat note between the generated probe beam and a local oscillator beam which is additionally frequency shifted by passing through an acousto-optic modulator (AOM), as shown in Fig. 2. The $\mathrm{x}$ axis in Fig. 3 refers to this beat-note frequency.

As can be seen from this figure, the width of the generated field is about $3 \mathrm{kHz}$ at a resolution bandwidth (RBW) of about $100 \mathrm{~Hz}$. Such a narrow width for the generated field is only possible when the decay processes involving optical excited states are completely absent in the sum-frequency generation. This is remarkable because the coupling field is on resonance with the $|2\rangle \rightarrow|3\rangle$ transition which has a natural decay width $\Gamma_{31}$ of about $5 \mathrm{MHz}$ due to the strong possibility for spontaneous decay into the frequency regime of the sum-frequency generation process. The fact that we have experimentally observed a much narrower linewidth is indicative of the suppression of one-photon optical resonance processes in our system. The width of $3 \mathrm{kHz}$ is very close to the $\Gamma_{21}$ value of $1 \mathrm{kHz}$, which is the meta-stable state decay width.

We now demonstrate that the sum-frequency generation satisfies a three-photon resonance $\delta_{\mathrm{p}}-\delta_{\mathrm{c}}-\delta_{\mu \mathrm{w}}=0$, which is demanded out of energy conservation when one coupling optical photon combines with a microwave drive photon to generate an optical probe photon. This is shown in Fig. 4(a). This plot is obtained by varying the detuning $\delta_{\mu \mathrm{w}}$ of the microwave field, keeping the detuning of coupling field $\delta_{\mathrm{c}}=0$.

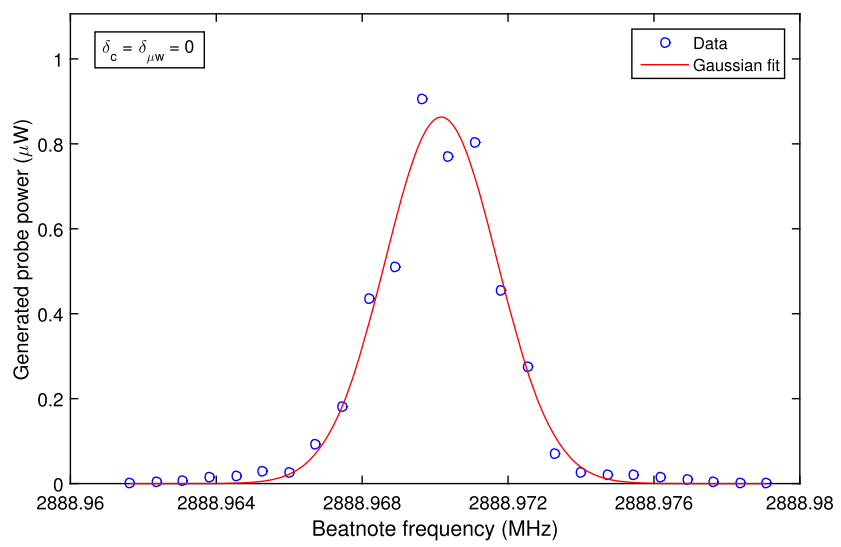

Fig. 3. Spectral width of the generated probe field is shown when both the coupling and the microwave fields are at resonance with their respective transitions. The experimental points are shown as circles with a Gaussian fit shown as a continuous line. The RBW was $100 \mathrm{~Hz}$ for this measurement. 

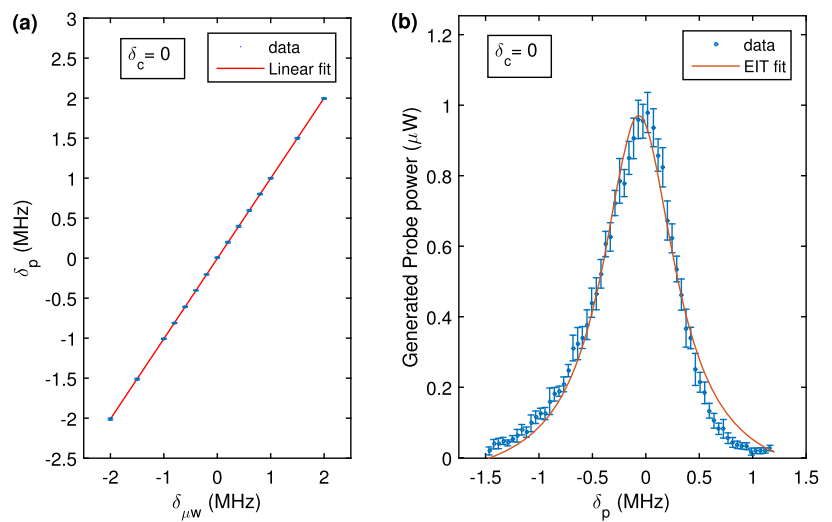

Fig. 4. (a) Detuning $\delta_{p}$ at which the maximum probe light generation is seen as a function of detuning $\delta_{\mu \mathrm{w}}$ of the microwave drive field with the coupling field on resonance with $\delta_{c}=0$. (b) Generated probe power as a function of probe field detuning $\delta_{p}$ when $\delta_{c}=0$. The values of $\delta_{p}$ are extracted from (a) for the corresponding values of microwave detuning $\delta_{\mu \mathrm{w}}$.

We see in this plot that the frequency of the maximum probe field generation denoted by $\delta_{\mathrm{p}}$ has a one-to-one scaling with the detuning of the microwave drive field. This is expected from the three-photon resonance equation with $\delta_{\mathrm{c}}=0$. The error bars in this plot denote the narrow range over which this condition is satisfied. Thus, this plot not only confirms the nonlinear frequency conversion, but also shows that a generation process occurs, even when the microwave drive field is nonresonant with its transition.

In order to confirm the suppression of one-photon optical processes in our generation scheme, we examined the possibility that our undepleted coupling beam and the generated probe beam are shelving the atom in a dark state due to the fulfillment of a two-photon Raman resonance condition. To verify this, we measure the power of the generated probe field with $\delta_{\mathrm{c}}=0$ with varied values of microwave detuning $\delta_{\mu \mathrm{w}}$. The corresponding probe field detunings for generation are obtained from Fig. 4(a). The result of these measurements is plotted in Fig. 4(b). Every point along this curve satisfies the three-photon resonance condition which is a necessary and sufficient condition for probe generation but, as can be seen in Fig. 4(b), the generated probe power does not remain constant and varies along this curve. We find that the probe beam has maximum power only at $\delta_{p}=\delta_{c}$. This establishes that the sum-frequency generation has maximum efficiency when a two-photon condition is also satisfied. This two-photon condition shelves the atom in a dark state, giving rise to an induced transparency effect [24] for the probe. Therefore uniquely for our system, the process of nonlinear generation of the probe light also minimizes its one-photon optical losses.

The solid line in Fig. 4(b) is a fit to our experimental data fitted with the formula [26]

$$
P_{\mathrm{p}}=C-\left(\frac{C_{+}^{2}}{\Gamma_{+}^{2}+\delta^{2}}-\frac{C_{-}^{2}}{\Gamma_{-}^{2}+\delta^{2}}\right) .
$$

This fit represents the fact the generated probe satisfies Fanointerference [27]. This formula decomposes the power of the generated probe $P_{\mathrm{p}}$ as a sum of two Lorentzians. The fact that we are measuring a transmitted beam is represented by subtracting the sum from an overall constant $\mathrm{C}$. The parameters of the Lorentzian, namely their widths $\left(\Gamma_{+}\right.$and $\left.\Gamma_{-}\right)$and amplitudes $\left(C_{+}\right.$and $\left.C_{-}\right)$, are obtained as numerical fit parameters to the experimental data. From the fitted values, we find the width $2 \Gamma_{\text {- }}$ which arises from Raman coherence to be about $825 \mathrm{kHz}$

We have measured the dependence of the generated probe power as a function of both the drive microwave field intensity and the coupling optical field intensity. As shown in Fig. 5(a), the generated probe power varies linearly with the input microwave intensity for small intensities and reaches saturation beyond an input intensity of about $4.4 \mathrm{~mW} / \mathrm{cm}^{2}$.

A similar trend is also seen in Fig. 5(b), where the generated probe power is plotted as a function of the input coupling intensity. We see that, beyond a coupling intensity of about $38 \mathrm{~mW} / \mathrm{cm}^{2}$, the generated probe power remains constant. In nonlinear wave generation processes, the linear regime indicates that the input coupling and drive fields are undepleted [28]. Beyond the linear regime, the coupling and microwave drive fields begin to be substantially absorbed. In this regime, the imaginary part of the nonlinear susceptibility cannot be ignored, resulting in saturation of the generated probe field.

The efficiency $\eta$ of probe generation is calculated using the definition [16]

$$
\eta=\frac{\frac{P_{p}}{h \nu_{p}}}{\frac{I_{\mu} A_{\mu}}{h \nu_{\mu}}},
$$

where $P_{p}$ is the generated probe power at frequency $\nu_{p}$, and $I_{\mu}$ is the input microwave intensity at frequency $\nu_{\mu}$. Here $A_{\mu}$ is the cross-sectional area of a microwave with intensity $I_{\mu}$ which interacts with the atoms. The maximum value for $\eta$ that is seen in our experiment is $2.6 \times 10^{-4} \%$.

The factors that affect the efficiency are the weakness of magnetic-dipole transition induced by the microwave field and the lower number density obtainable with room temperature atomic vapors.

We have explicitly confirmed the coherent nature of our generation process by the following experiment. We derive a local oscillator beam by sending a part of the coupling beam to an electro-optic modulator (EOM). The EOM is driven
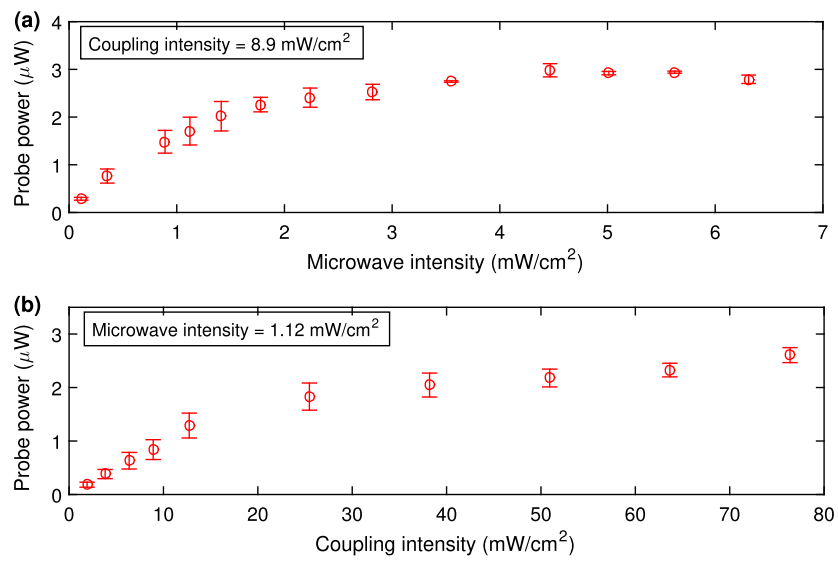

Fig. 5. (a) Generated optical probe power as a function of the input microwave intensity at constant coupling field intensity. (b) Generated optical probe power as a function of the coupling field intensity at a constant microwave field intensity. 


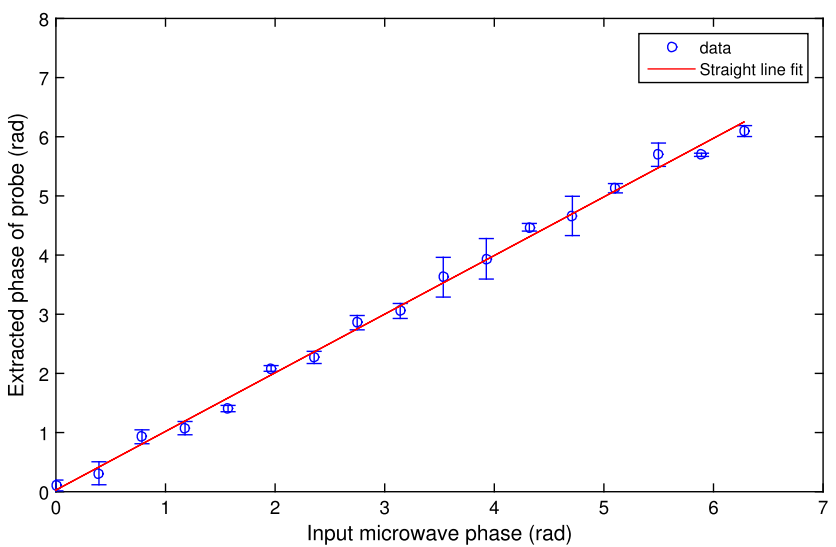

Fig. 6. Extracted optical phase of the generated probe field as a function of the input phase imposed on the microwave field.

by the same microwave source at $3.0357 \mathrm{GHz}$ which is used to drive our microwave cavity. The EOM generates two sidebands at $\omega_{c} \pm 3.0357 \mathrm{GHz}$ frequencies. We now mix the generated probe frequency with the frequencies generated by the EOM. We detect the beat note using a spectrum analyzer.

We now introduce known phase shifts to the microwave that are feeding our cavity, keeping its power constant through an external microwave phase shifter. We then measure the changing power at our heterodyne measurement frequency which results from changes in the generated optical phase. We then extract the optical phase from this data. This is given in Fig. 6. From this figure, it is clear that the probe phase changes commensurately with a change in the microwave phase, proving the coherent nature of probe field generation.

The three-wave mixing coherent generation described in this Letter, to the best of our knowledge, is the first such observation reported so far from atomic systems in any frequency regime. Such an observation has not been possible because of the absence of any three-wave processes for electric-dipole mediated nonlinear generation in centro-symmetric atomic systems. In our system, because of the participation of an induced magnetic dipole, the symmetry is broken, allowing for a three-wave process. This significantly reduces the number of input beams required and enormously simplifies the experimental requirements. To put this in context, the only experimental demonstration of coherent generation between microwave and optical frequency regimes from atomic systems utilized a six-wave mixing process and required ultra-cold atomic samples [16].

Despite the low efficiency of our conversion process due to the magnetic-dipole interaction, our coherent microwave-tooptical conversion has the potential for high fidelity signal transfer between these two frequency regimes. This arises from the fact that the two-photon resonance in our scheme prevents decoherence through spontaneous emission by shelving the atom in dark states which are the very states used traditionally as quantum memory states. Hence, our scheme is ideally suited for the transfer of quantum information, where the fidelity of transfer is essentially limited by optical quantum memory [11] and not by loss of signal strength [25].

Funding. Department of Science and Technology, India..

\section{REFERENCES}

1. G. T. Ao, S. Tanzilli, H. Zbinden, N. Gisin, T. Aellen, M. Giovannini, and J. Faist, Opt. Lett. 31, 1094 (2006).

2. M. J. Khan, J. C. Chen, and S. Kaushik, Opt. Lett. 32, 3248 (2007)

3. D. V. Strekalov, A. A. Savchenkov, A. B. Matsko, and N. Yu, Opt. Lett. 34, 713 (2009).

4. G. K. Gopalakrishnan, W. K. Burns, and C. H. Bulmer, IEEE Trans. Microw. Theory Tech. 41, 2383 (1993).

5. Z.-L. Xiang, S. Ashhab, J. Q. You, and F. Nori, Rev. Mod. Phys. 85, 623 (2013).

6. D. V. Strekalov, H. G. L. Schwefel, A. A. Savchenkov, A. B. Matsko, L. J. Wang, and N. Yu, Phys. Rev. A 80, 033810 (2009).

7. A. Rueda, F. Sedlmeir, M. C. Collodo, U. Vogl, B. Stiller, G. Schunk, D. V. Strekalov, C. Marquardt, J. M. Fink, O. Painter, G. Leuchs, and H. G. L. Schwefel, Optica 3, 597 (2016).

8. J. Bochmann, A. Vainsencher, D. D. Awschalom, and A. N. Cleland, Nat. Phys. 9, 712 (2013).

9. R. W. Andrews, R. W. Peterson, T. P. Purdy, K. Cicak, R. W. Simmonds, C. A. Regal, and K. W. Lehnert, Nat. Phys. 10, 321 (2014).

10. C. O'Brien, N. Lauk, S. Blum, G. Morigi, and M. Fleischhauer, Phys. Rev. Lett. 113, 063603 (2014).

11. S. Blum, C. O'Brien, N. Lauk, P. Bushev, M. Fleischhauer, and G. Morigi, Phys. Rev. A 91, 033834 (2015).

12. M. Hafezi, Z. Kim, S. L. Rolston, L. A. Orozco, B. Lev, and J. M. Taylor, Phys. Rev. A 85, 020302 (2012).

13. M. Kiffner, A. Feizpour, K. T. Kaczmarek, D. Jaksch, and J. Nunn, New J. Phys. 18, 093030 (2016).

14. B. T. Gard, K. Jacobs, R. McDermott, and M. Saffman, Phys. Rev. A 96, 013833 (2017).

15. L. A. Williamson, Y.-H. Chen, and J. J. Longdell, Phys. Rev. Lett. 113, 203601 (2014).

16. J. Han, T. Vogt, C. Gross, D. Jaksch, M. Kiffner, and W. Li, Phys. Rev. Lett. 120, 093201 (2018).

17. Z. Qin, L. Cao, H. Wang, A. M. Marino, W. Zhang, and J. Jing, Phys. Rev. Lett. 113, 023602 (2014).

18. V. Boyer, A. M. Marino, and P. D. Lett, Phys. Rev. Lett. 100, 143601 (2008).

19. M. O. Scully and M. Fleischhaurer, Science 263, 337 (1994).

20. S. E. Harris, J. E. Field, and A. Imamoğlu, Phys. Rev. Lett. 64, 1107 (1990).

21. T. Meijer, J. White, B. Smeets, M. Jeppesen, and R. Scholten, Opt. Lett. 31, 1002 (2006).

22. A. M. Akulshin, R. J. McLean, A. I. Sidorov, and P. Hannaford, Opt. Express 17, 22861 (2009).

23. A. Akulshin, D. Budker, and R. McLean, Opt. Lett. 39, 845 (2014).

24. S. E. Harris, Phys. Rev. Lett. 62, 1033 (1989).

25. A. Fedrizzi, R. Ursin, T. Herbst, M. Nespoli, R. Prevedel, T. Scheidl, F. Tiefenbacher, T. Jennewein, and A. Zeilinger, Nat. Phys. 5, 389 (2009).

26. P. M. Anisimov, J. P. Dowling, and B. C. Sanders, Phys. Rev. Lett. 107, 163604 (2011).

27. P. Anisimov and O. Kocharovskaya, J. Mod. Opt. 55, 3159 (2008).

28. R. W. Boyd, Nonlinear Optics, 3rd ed. (Academic, 2008). 